\title{
Eigenvalue Asymptotics in a Twisted Waveguide
}

\author{
October 31, 2018
}

\author{
Philippe Briet, Hynek Kovařík, Georgi Raikov, Eric Soccorsi
}

\begin{abstract}
We consider a twisted quantum wave guide i.e. a domain of the form $\Omega_{\theta}:=$ $r_{\theta} \omega \times \mathbb{R}$ where $\omega$ is a connected open and bounded subset of $\mathbb{R}^{2}$ and $r_{\theta}=r_{\theta}\left(x_{3}\right)$ is a rotation by the angle $\theta\left(x_{3}\right)$ depending on the longitudinal variable $x_{3}$. We are interested in the spectral analysis of the Dirichlet Laplacian $H$ acting in $\Omega_{\theta}$. We suppose that the derivative $\dot{\theta}$ of the rotation angle can be written as $\dot{\theta}\left(x_{3}\right)=$ $\beta-\varepsilon\left(x_{3}\right)$ with a positive constant $\beta$ and $\varepsilon\left(x_{3}\right) \sim L\left|x_{3}\right|^{-\alpha},\left|x_{3}\right| \rightarrow \infty$. We show that if $L>0$ and $\alpha \in(0,2)$, or if $L>L_{0}>0$ and $\alpha=2$, then there is an infinite sequence of discrete eigenvalues lying below the infimum of the essential spectrum of $H$, and obtain the main asymptotic term of this sequence.
\end{abstract}

AMS 2000 Mathematics Subject Classification: 35J10, 81Q10, 35P20

Keywords: Waveguides, eigenvalue asymptotics, Schrödinger operators

\section{Introduction}

In this paper we deal with the spectrum of the Dirichlet Laplacian in the twisted waveguide $\Omega_{\theta}:=r_{\theta} \omega \times \mathbb{R}$ where $\omega$ is a connected open and bounded subset of $\mathbb{R}^{2}$ with sufficiently regular boundary, and $r_{\theta}=r_{\theta}\left(x_{3}\right)$ is a rotation by the angle $\theta\left(x_{3}\right)$ depending on the longitudinal variable $x_{3}$.

If the twisting of $\Omega_{\theta}$ is only local, then it does not affect the essential spectrum of the Dirichlet Laplacian. However, it does stabilise the discrete spectrum against possible negative perturbations. Namely, it has been found out recently that, provided $\omega$ is not rotationally symmetric, the local twisting of $\Omega$ can be interpreted as a kind of a repulsive perturbation, see [10, 13]. This has several consequences such as the absence of weakly coupled bound states of Schrödinger operators in twisted waveguides, see [13], [21.

From this point of view the effect of a local twisting of a three-dimensional waveguide is similar to the effect of a local magnetic field in a two-dimensional waveguide, [12]. Moreover, if the twisting is not local but constant, then it even increases the infimum of the essential spectrum of the Dirichlet Laplacian, see [14]. This is again reminiscent of the situation in two-dimensional strips with constant magnetic field, see [11, 15]. On 
the other hand, any local decrease of a constant twisting will induce at least one bound state of the corresponding Dirichlet Laplacian, see [14].

In the present paper we study in more detail the properties of these bound states induced by twisting. It is well known that the Schrödinger operator with a slowly decaying potential possesses infinitely many bound states, and that the asymptotic distribution of these bound states depends on the behaviour of the potential at infinity, see e.g. [22, 24]. Our aim is to obtain analogous asymptotic results for the bound states which are not induced by an external potential, but by the twisting of the waveguide.

We start with the analysis of the unperturbed operator, which corresponds to a constant twisting. This operator is translationally invariant in the longitudinal direction and therefore allows a fiber decomposition with fiber operators which have purely discrete spectrum, see Subsection 2.2. As in the model with constant magnetic field, [11, 15], we first analyze the structure of the corresponding band functions. In particular, we prove the existence of an effective mass at the bottom of the spectrum of the unperturbed operator, see Theorem 3.1. Then we show that if the constant twisting is perturbed by the function $\varepsilon=\varepsilon\left(x_{3}\right)$ which decays slowly enough at infinity, then the resulting operator has infinitely many discrete bound states accumulating from below at the infimum of the essential spectrum. Finally, we employ the approach of [23] in order to study the rate of accumulation of these bound states. Roughly speaking, our main result, Theorem 4.4, says that the rate of accumulation is determined by the rate of decay of $\varepsilon$ at infinity, and by the geometry of the cross-section $\omega$.

\section{The Dirichlet Laplacian}

\subsection{The general case}

Let $\omega$ be a bounded domain in $\mathbb{R}^{2}$ with $C^{2}$-boundary. Furthermore, we suppose that $\omega$ contains the origin of $\mathbb{R}^{2}$. Set $\Omega=\omega \times \mathbb{R}$. For $\mathbf{x}=\left(x_{1}, x_{2}, x_{3}\right) \in \Omega$ we write $\mathbf{x}=\left(x_{t}, x_{3}\right)$ with $x_{t}=\left(x_{1}, x_{2}\right)$. Assume that $\theta \in C^{1}(\mathbb{R})$ and its derivative is bounded on $\mathbb{R}$. Define the twisted domain

$$
\Omega_{\theta}=\left\{r_{\theta}\left(x_{3}\right)(\mathbf{x}), \mathbf{x} \in \Omega\right\}
$$

where

$$
r_{\theta}\left(x_{3}\right)=\left(\begin{array}{ccc}
\cos \theta\left(x_{3}\right) & \sin \theta\left(x_{3}\right) & 0 \\
-\sin \theta\left(x_{3}\right) & \cos \theta\left(x_{3}\right) & 0 \\
0 & 0 & 1
\end{array}\right) .
$$

We consider the Dirichlet Laplacian $-\Delta^{D}$ in $\mathrm{L}^{2}\left(\Omega_{\theta}\right)$, i.e. the self-adjoint operator generated in $\mathrm{L}^{2}\left(\Omega_{\theta}\right)$ by the closed quadratic form

$$
\tilde{\mathcal{Q}}_{\theta}[f]=\int_{\Omega_{\theta}}|\nabla f(\mathbf{x})|^{2} d \mathbf{x}, \quad f \in \mathrm{D}\left(\tilde{\mathcal{Q}}_{\theta}\right)=\mathrm{H}_{0}^{1}\left(\Omega_{\theta}\right) .
$$


Here $\mathrm{H}_{0}^{1}\left(\Omega_{\theta}\right)$ denotes, as usual, the closure of $C_{0}^{\infty}\left(\Omega_{\theta}\right)$ in the topology of the standard first-order Sobolev space $\mathrm{H}^{1}\left(\Omega_{\theta}\right)$. Introduce the transform

$$
(\mathcal{U} f)(\mathbf{x})=f\left(r_{\theta}\left(x_{3}\right)(\mathbf{x})\right), \quad \mathbf{x} \in \Omega, \quad f \in \mathrm{L}^{2}\left(\Omega_{\theta}\right) .
$$

It is easy to see that $\mathcal{U}$ is a unitary operator from $\mathrm{L}^{2}\left(\Omega_{\theta}\right)$ onto $\mathrm{L}^{2}(\Omega)$. Note also that $\mathcal{U}\left(\mathrm{H}_{0}^{1}\left(\Omega_{\theta}\right)\right)=\mathrm{H}_{0}^{1}(\Omega)$. Set

$$
\nabla_{t}:=\left(\partial_{1}, \partial_{2}\right)^{T}, \quad \Delta_{t}:=\partial_{1}^{2}+\partial_{2}^{2}, \quad \partial_{\varphi}:=x_{1} \partial_{2}-x_{2} \partial_{1} .
$$

Denote by $\dot{\theta}$ the derivative of $\theta$. Define the operator $H_{\dot{\theta}}$ as the self-adjoint operator generated in $\mathrm{L}^{2}(\Omega)$ by the closed quadratic form

$$
\mathcal{Q}_{\dot{\theta}}[f]:=\tilde{\mathcal{Q}}_{\theta}\left[\mathcal{U}^{-1} f\right]=\int_{\Omega}\left(\left|\nabla_{t} f\right|^{2}+\left|\dot{\theta}\left(x_{3}\right) \partial_{\varphi} f+\partial_{3} f\right|^{2}\right) d \mathbf{x}, \quad f \in \mathrm{H}_{0}^{1}(\Omega)=: D\left(H_{\dot{\theta}}^{1 / 2}\right) .
$$

Evidently, $H_{\dot{\theta}}=\mathcal{U}\left(-\Delta^{D}\right) \mathcal{U}^{-1}$. By a straightforward computation we find out that $H_{\dot{\theta}}$ acts on its domain as

$$
H_{\dot{\theta}}=-\Delta_{t}-\left(\dot{\theta}\left(x_{3}\right) \partial_{\varphi}+\partial_{3}\right)^{2} .
$$

Moreover, since $\omega$ is a bounded domain, and we impose Dirichlet boundary conditions, the operator $H_{\dot{\theta}}$ is strictly positive, and hence boundedly invertible in $\mathrm{L}^{2}(\Omega)$.

Remark 2.1. If $\omega$ is a disk centered at the origin, then $\Omega_{\theta}=\Omega$ for any twisting $\theta$, and the operator $H_{\dot{\theta}}$ is unitarily equivalent to $H_{0}$. Note that $H_{0}$ has purely absolutely continuous spectrum (see e.g. (2.4) below).

\subsection{Constant twisting}

In this subsection we assume that the twisting is constant, i.e. there exists $\beta \in \mathbb{R}$ such that $\dot{\theta}\left(x_{3}\right)=\beta$ for every $x_{3} \in \mathbb{R}$.

Let $\mathcal{F}$ be the partial Fourier transform with respect to $x_{3}$, i.e.

$$
(\mathcal{F} u)\left(x_{t}, p\right):=\frac{1}{\sqrt{2 \pi}} \int_{\mathbb{R}} e^{-i p x_{3}} u\left(x_{t}, x_{3}\right) d x_{3}, \quad\left(x_{t}, p\right) \in \omega \times \mathbb{R} .
$$

Due to the translational invariance in the $x_{3}$-direction, the operator $\hat{H}_{\beta}:=\mathcal{F} H_{\beta} \mathcal{F}^{*}$ admits a direct integral decomposition

$$
\hat{H}_{\beta}:=\int_{\mathbb{R}}^{\oplus} h_{\beta}(p) d p
$$

where

$$
h_{\beta}(p):=-\Delta_{t}-\left(\beta \partial_{\varphi}+i p\right)^{2}, \quad p \in \mathbb{R}
$$


is the self-adjoint operator generated in $\mathrm{L}^{2}(\omega)$ by the closed quadratic form

$$
q_{p}[u]=q_{p, \beta}[u]:=\int_{\omega}\left(\left|\nabla_{t} u\left(x_{t}\right)\right|^{2}+\left|\left(i \beta \partial_{\varphi}-p\right) u\left(x_{t}\right)\right|^{2}\right) d x_{t}, \quad u \in \mathrm{H}_{0}^{1}(\omega) .
$$

Evidently, for each $p \in \mathbb{R}$ the quadratic form $q_{p}$ induces an equivalent norm on $\mathrm{H}_{0}^{1}(\omega)$. Moreover, since the quadratic form Re $i \int_{\omega} \partial_{\varphi} u \bar{u} d x_{t}, u \in \mathrm{H}_{0}^{1}(\omega)$, is relatively bounded with respect to the quadratic form $q_{0, \beta}[u]$ with zero relative bound, we find that $h_{\beta}(p)$, $p \in \mathbb{C}$, is a Kato analytic family of type B (see [18] or [24, Theorem XII.10]).

Further, since $\omega$ is a bounded domain with $C^{2}$-boundary, the domain of the operator $h_{\beta}(p)$ is $\mathrm{H}^{2}(\omega) \cap \mathrm{H}_{0}^{1}(\omega)$ for each $p \in \mathbb{R}$ (see e.g. [2]).

Next, by the compactness of the embedding $\mathrm{H}_{0}^{1}(\omega) \hookrightarrow \mathrm{L}^{2}(\omega)$, the spectrum of the operator $h_{\beta}(p)$ is purely discrete. Let $\left\{E_{j}(p)\right\}_{j=1}^{\infty}=\left\{E_{j}(p, \beta)\right\}_{j=1}^{\infty}$ be the non-decreasing sequence of the eigenvalues of $h_{\beta}(p), p \in \mathbb{R}$. Since $h_{\beta}(p)$ is a Kato analytic family, the functions $\mathbb{R} \ni p \mapsto E_{j}(p) \in(0, \infty), j \in \mathbb{N}$, are continuous piece-wise analytic functions (see e.g. [24, Theorem XII.12]). The mini-max principle easily yields

$$
E_{j}(p)=p^{2}(1+o(1)), \quad p \rightarrow \pm \infty .
$$

Therefore, the general theory of analytically fibred operators (see e.g. [24, Section XIII.16]) implies that the spectrum of the operator $H_{\beta}$ is purely absolutely continuous. In summary, we have

$$
\sigma\left(H_{\beta}\right)=\sigma_{\mathrm{ac}}\left(H_{\beta}\right)=\cup_{j \in \mathbb{N}} E_{j}(\mathbb{R})=[\mathcal{E}, \infty),
$$

with

$$
\mathcal{E}=\mathcal{E}(\beta):=\min _{p \in \mathbb{R}} E_{1}(p, \beta) .
$$

Furthermore, for $p \in \mathbb{R}$ introduce a family $\left\{\psi_{j}\left(x_{t} ; p\right)\right\}_{j=1}^{\infty}$ of eigenfunctions of the operator $h(p)$ which satisfy

$$
\left(h(p) \psi_{j}\right)\left(x_{t} ; p\right)=E_{j}(p) \psi_{j}\left(x_{t} ; p\right), \quad x_{t} \in \omega
$$

and form an orthonormal basis in $\mathrm{L}^{2}(\omega)$. By the embedding $\mathrm{H}^{2}(\omega) \hookrightarrow C^{l}(\bar{\omega})$ with $l \in[0,1)$, we have $\psi_{j}(\cdot ; p) \in C^{l}(\bar{\omega}), j \in \mathbb{N}$; in particular, the eigenfunctions $\psi_{j}(\cdot ; p)$ are continuous and bounded on $\bar{\omega}$. Moreover, since the operator $h_{\beta}(p)$ is strongly elliptic with coefficients in $C^{\infty}(\omega)$, we have $\psi_{j}(\cdot ; p) \in C^{\infty}(\omega), j \in \mathbb{N}$ (see e.g. [2], [24]).

Next, note that $h_{\beta}(0)$ is a strongly elliptic operator with real coefficients. Therefore, $E_{1}(0, \beta)$ is a simple eigenvalue of $h_{\beta}(0)$. Hence, we can choose $\psi_{1}(\cdot ; 0)$ to be real and positive in $\omega$ (see e.g. [16, Theorem 8.38]). Moreover, there exists $\delta>0$ such that the eigenvalue $E_{1}(p)$ is simple for $p \in[-\delta, \delta]$, and therefore the mapping $[-\delta, \delta] \ni p \mapsto$ $E_{1}(p) \in(0, \infty)$ is analytic. Similarly, $\psi_{1}(\cdot ; p)$ could be chosen so that the mapping $[-\delta, \delta] \ni p \mapsto \psi_{1}(\cdot ; p) \in \mathrm{H}^{2}(\omega)$ is analytic.

At the end of this section, we establish for further references a criterion which guarantees that $\omega$ is a disk centered at the origin. 
Proposition 2.2. Let $\omega \subset \mathbb{R}^{2}$ be a bounded domain with $C^{2}$-boundary $\partial \omega$. Assume that $\omega$ contains the origin. Then $\omega$ is a disk centered at the origin if and only if

$$
\left\|\partial_{\varphi} \psi_{1}(\cdot ; 0)\right\|_{L^{2}(\omega)}=0 .
$$

Proof. For brevity set $\psi\left(x_{t}\right)=\psi_{1}\left(x_{t} ; 0\right), x_{t} \in \omega$. Note that (2.7) implies $\partial_{\varphi} \psi=0$ identically in $\omega$ since $\psi \in C^{\infty}(\omega)$.

(i) Assume that (2.7) holds true. Since $\psi$ is radial, it satisfies the equation

$$
\left(\Delta_{t}+\mathcal{E}\right) \psi=0
$$

in $\omega$ (see (2.3)). Pick $\epsilon>0$ such that the open disk $B_{\epsilon}:=\left\{x_{t} \in \mathbb{R}^{2}|| x_{t} \mid<\epsilon\right\}$ is contained in $\omega$. Since $\psi$ is radial, regular, and satisfies (2.8) in $B_{\epsilon}$ we find that

$$
\psi\left(x_{t}\right)=\tilde{\psi}\left(x_{t}\right), \quad x_{t} \in B_{\varepsilon},
$$

with $\tilde{\psi}\left(x_{t}\right)=c J_{0}\left(\mathcal{E}^{1 / 2}\left|x_{t}\right|\right), x_{t} \in \mathbb{R}^{2}$, where $J_{0}$ is the zeroth-order Bessel function (see e.g. [1, Section 9.1]), and $c \neq 0$; if $c=0$, then the unique continuation principle (see e.g. [17]) would imply that $\psi=0$ identically in $\omega$ which contradicts the fact that $\psi$ is an eigenfunction. Note that

$$
\left(\Delta_{t}+\mathcal{E}\right) \tilde{\psi}=0
$$

in $\mathbb{R}^{2}$. Comparing (2.8) with (2.10), and bearing in mind the unique continuation principle, we find that (2.9) holds for all $x_{t} \in \omega$.

Let now $\left\{\mathcal{C}_{\alpha}\right\}$ be the set of the connected components of $\partial \omega$. Fix $\alpha$ and introduce the function $\varrho_{\alpha}$ by $\mathcal{C}_{\alpha} \ni x_{t} \mapsto \varrho_{\alpha}\left(x_{t}\right):=\left|x_{t}\right| \in(0, \infty)$. Set $\mathcal{I}_{\alpha}:=\varrho_{\alpha}\left(\mathcal{C}_{\alpha}\right)$. Since $\mathcal{C}_{\alpha}$ is connected, and $\varrho_{\alpha}$ is continuous, $\mathcal{I}_{\alpha}$ should be connected too, i.e. $\mathcal{I}_{\alpha}$ is a one-point set, or a bounded interval of positive length. Due to the Dirichlet boundary conditions, we have $\psi\left(x_{t}\right)=0$ for all $x_{t} \in \mathcal{C}_{\alpha}$, i.e. $J_{0}\left(\mathcal{E}^{1 / 2} r\right)=0$ for all $r \in \mathcal{I}_{\alpha}$. Since $J_{0}$ has at most a finite number of zeros on any bounded interval, all $\mathcal{I}_{\alpha}$ are one-point sets, i.e. all $\mathcal{C}_{\alpha}$ are arcs of circles centered at the origin. Since $\partial \omega \in C^{2}$, all $\mathcal{C}_{\alpha}$ are circles. Since that $\omega$ is connected and contains the origin, it is a disk centered at the origin.

(ii) Assume now that $\omega$ is a disk of radius $R \in(0, \infty)$, centered at the origin. Passing to polar coordinates $(r, \varphi)$, and writing

$$
u(r \cos \varphi, r \sin \varphi)=\frac{1}{\sqrt{2 \pi}} \sum_{m \in \mathbb{Z}} e^{i m \varphi} u_{m}(r), \quad u \in D\left(h_{\beta}(0)\right),
$$

we find that $h_{\beta}(0)$ is unitarily equivalent to $\oplus_{m \in \mathbb{Z}} \mathcal{H}_{m}$, where $\mathcal{H}_{m}, m \in \mathbb{Z}$, is the operator generated in $\mathrm{L}^{2}((0, R) ; r d r)$ by the closure of the quadratic form

$$
\int_{0}^{R}\left(\left|u_{m}^{\prime}(r)\right|^{2}+m^{2}\left(r^{-2}+\beta^{2}\right)\left|u_{m}(r)\right|^{2}\right) r d r, \quad u_{m} \in C_{0}^{\infty}(0, R) .
$$

The spectra of all the operators $\mathcal{H}_{m}$ are discrete. Since $\mathcal{E}=\inf _{m \in \mathbb{Z}} \inf \sigma\left(\mathcal{H}_{m}\right)$, and $\mathcal{H}_{m} \geq \mathcal{H}_{0}$ for all $m \in \mathbb{Z}$, we find that $\mathcal{E}$ coincides with the first eigenvalue of $\mathcal{H}_{0}$, which is simple. Let $\tilde{\psi}$ be the real-valued eigenfunction of $\mathcal{H}_{0}$ which satisfies $\mathcal{H}_{0} \tilde{\psi}=\mathcal{E} \tilde{\psi}$ and $\int_{0}^{R} \tilde{\psi}(r)^{2} r d r=1$. Since $\mathcal{E}$ is a simple eigenvalue of $h_{\beta}(0)$, it is not an eigenvalue of any $\mathcal{H}_{m}$ with $m \neq 0$. Therefore, $\psi\left(x_{t}\right)=\tilde{\psi}\left(\left|x_{t}\right|\right), x_{t} \in \omega$, i.e. $\psi$ is radial. 


\section{Existence of an Effective Mass at the Origin}

In this section assume that $\dot{\theta}$ is equal to the constant $\beta \in \mathbb{R}$, and investigate the properties of the first band function $E_{1}(p), p \in \mathbb{R}$. We show that $E_{1}(0)$ attains its minimum value $\mathcal{E}$ at $p=0$ and only at $p=0$, and the minimum is non-degenerate, i.e. $E_{1}^{\prime \prime}(0)>0$. In this case, following a terminology established in the solid-state physics (see e.g. [19]), we say that there exists an effective mass at the origin. The existence of an effective mass at the bottom of the absolutely continuous spectrum is an important problem in the spectral analysis of many operators of the mathematical physics (see e.g. [19, 23, 9, 4]).

For $\beta \in \mathbb{R}$ define

$$
\epsilon_{\omega}(\beta):=\frac{\beta^{2} C_{\omega}}{1+\beta^{2} C_{\omega}}
$$

where

$$
C_{\omega}:=\sup _{x_{t} \in \omega}\left(x_{1}^{2}+x_{2}^{2}\right) .
$$

Theorem 3.1. Let $\beta \in \mathbb{R}$. Then

$$
E_{1}(0, \beta)+\left(1-\epsilon_{\omega}(\beta)\right) p^{2} \leq E_{1}(p, \beta) \leq E_{1}(0, \beta)+p^{2}, \quad p \in \mathbb{R} .
$$

To prove the Theorem 3.1 we need the following technical result:

Lemma 3.2. For all $p, \beta \in \mathbb{R}$ we have

$$
E_{1}(p, \beta)=E_{1}(0, \beta)+\inf _{0 \neq u \in C_{0}^{\infty}(\omega)} \frac{\int_{\omega} \psi_{1}^{2}\left(\left|\nabla_{t} u\right|^{2}+\left|i \beta \partial_{\varphi} u-p u\right|^{2}\right) d x_{t}}{\int_{\omega} \psi_{1}^{2}|u|^{2} d x_{t}}
$$

where $\psi_{1}=\psi_{1}(\cdot ; 0)$.

Proof. The starting point of the proof is the mini-max principle

$$
E_{1}(p, \beta)=\inf _{0 \neq v \in C_{0}^{\infty}(\omega)} \frac{\int_{\omega}\left(\left|\nabla_{t} v\right|^{2}+\left|i \beta \partial_{\varphi} v-p v\right|^{2}\right) d x_{t}}{\int_{\omega}|v|^{2} d x_{t}} .
$$

Change the functional variable $v=\psi_{1} u$. Note that $\psi_{1}^{-1} C_{0}^{\infty}(\omega)=C_{0}^{\infty}(\omega)$. Integrating by parts, and bearing in mind that the function $\psi_{1}(\cdot ; 0)$ is real-valued, we easily get

$$
\begin{gathered}
\int_{\omega}\left(\left|\nabla_{t} v\right|^{2}+\left|i \beta \partial_{\varphi} v-p v\right|^{2}\right) d x_{t}= \\
\int_{\omega} \psi_{1}^{2}\left(\left|\nabla_{t} u\right|^{2}+\left|i \beta \partial_{\varphi} u-p u\right|^{2}\right) d x_{t}+\int_{\omega} \psi_{1}\left(-\Delta_{t} \psi_{1}-\beta^{2} \partial_{\varphi}^{2} \psi_{1}\right)|u|^{2} d x_{t} .
\end{gathered}
$$

Taking into account the eigenvalue equation

$$
-\Delta_{t} \psi_{1}-\beta^{2} \partial_{\varphi}^{2} \psi_{1}=E_{1}(0, \beta) \psi_{1}
$$

we find that (3.5) and (3.6) imply (3.4). 
Proof of Theorem 3.1. We first prove the lower bound. For $\beta=0$ it follows from the Lemma 3.2 and

$$
\int_{\omega} \psi_{1}^{2}\left(\left|\nabla_{t} u\right|^{2}+p^{2}|u|^{2}\right) d x_{t} \geq p^{2} \int_{\omega} \psi_{1}^{2}|u|^{2} d x_{t}, \quad u \in C_{0}^{\infty}(\omega) .
$$

Let us now consider the case $\beta \neq 0$. Pick $\eta \in(0,1)$. Then we have

$$
\begin{gathered}
\int_{\omega} \psi_{1}^{2}\left(\left|\nabla_{t} u\right|^{2}+\left|i \beta \partial_{\varphi} u-p u\right|^{2}\right) d x_{t} \geq \\
\int_{\omega} \psi_{1}^{2}\left(\left|\nabla_{t} u\right|^{2}+\beta^{2}\left(1-\eta^{-1}\right)\left|\partial_{\varphi} u\right|^{2}+(1-\eta) p^{2}|u|^{2}\right) d x_{t} \geq \\
\left(1+\beta^{2}\left(1-\eta^{-1}\right) C_{\omega}\right) \int_{\omega} \psi_{1}^{2}\left|\nabla_{t} u\right|^{2} d x_{t}+(1-\eta) p^{2} \int_{\omega} \psi_{1}^{2}|u|^{2} d x_{t}, \quad u \in C_{0}^{\infty}(\omega),
\end{gathered}
$$

the constant $C_{\omega}$ being defined in (3.2). Choose $\eta=\epsilon_{\omega} \in(0,1)$, the constant $\epsilon_{\omega}$ being defined in (3.1). Then (3.7) implies

$$
\int_{\omega} \psi_{1}^{2}\left(\left|\nabla_{t} u\right|^{2}+\left|i \beta \partial_{\varphi} u-p u\right|^{2}\right) d x_{t} \geq\left(1-\epsilon_{\omega}\right) p^{2} \int_{\omega} \psi_{1}^{2}|u|^{2} d x_{t}
$$

and the lower bound in (3.3) follows from Lemma 3.2.

By the mini-max principle (3.5), and the fact that $\psi_{1}$ is real-valued, we get

$$
E_{1}(p, \beta) \leq \int_{\omega}\left(\left|\nabla_{t} \psi_{1}\right|^{2}+\left|i \beta \partial_{\varphi} \psi_{1}-p \psi_{1}\right|^{2}\right) d x_{t}=E_{1}(0, \beta)+p^{2}
$$

which gives the upper bound in (3.3).

Since $\epsilon_{\omega}(\beta)<1$, and $E_{1}(p, \beta)$ depends analytically on $p$ near the origin, Theorem 3.1 entails the following

Corollary 3.3. Let $\beta \in \mathbb{R}$. Then we have $\partial_{p} E_{1}(0, \beta)=0$ and

$$
\mu=\mu(\beta):=\frac{1}{2} \partial_{p}^{2} E_{1}(0, \beta)>0,
$$

i.e. at the origin there exists an effective mass. As a corollary,

$$
E_{1}(p, \beta)=E_{1}(0, \beta)+\mu(\beta) p^{2}+O\left(p^{3}\right), \quad p \rightarrow 0 .
$$

Moreover, for each $p \in \mathbb{R}, p \neq 0$, and $\beta \in \mathbb{R}$ we have $E_{1}(p, \beta)>E_{1}(0, \beta)$. 


\section{Eigenvalue Asymptotics for the Dirichlet Lapla- cian with Non-Constant Twisting}

\subsection{Perturbation of the twisting}

In this section the constant parameter $\beta>0$ is fixed, and we now consider the case where the derivative of the twisting is given by

$$
\dot{\theta}\left(x_{3}\right)=\beta-\varepsilon\left(x_{3}\right),
$$

where the perturbation $\varepsilon \in \mathrm{L}^{\infty}(\mathbb{R})$ satisfies $\varepsilon(x) \geq 0, x \in \mathbb{R}$, and $\lim _{x \rightarrow \pm \infty} \varepsilon(x)=0$. By (2.2), the operator $H_{\beta-\varepsilon}$ can be written as

$$
H_{\beta-\varepsilon}=H_{\beta}+W_{\varepsilon, \beta}
$$

where

$$
W_{\varepsilon, \beta}:=2 \beta \varepsilon \partial_{\varphi}^{2}+\partial_{\varphi} \varepsilon \partial_{3}+\partial_{3} \varepsilon \partial_{\varphi}-\varepsilon^{2} \partial_{\varphi}^{2}=-2 \beta G_{0}^{*} G_{0}-G_{0}^{*} G_{1}-G_{1}^{*} G_{0}+G_{2}^{*} G_{2},
$$

and

$$
G_{0}:=\varepsilon^{1 / 2} \partial_{\varphi}, \quad G_{1}:=\varepsilon^{1 / 2} \partial_{3}, \quad G_{2}:=\varepsilon \partial_{\varphi},
$$

are the operators defined on $D\left(H_{\beta}^{1 / 2}\right)=\mathrm{H}_{0}^{1}(\Omega)$. Evidently, the operators $G_{j} H_{\beta}^{-1 / 2}$, $j=0,1,2$, are bounded in $\mathrm{L}^{2}(\Omega)$. Due to the Sobolev embedding theorems and the fact that $\varepsilon(x) \rightarrow 0$ as $|x| \rightarrow \infty$, it is easy to see that the operators $G_{j} H_{\beta}^{-1}, j=$ $0,1,2$, are compact in $\mathrm{L}^{2}(\Omega)$. Therefore, the operator $H_{\beta}^{-1 / 2} W_{\varepsilon, \beta} H_{\beta}^{-1}$ is compact. Since the operators $H_{\beta-\varepsilon}^{-1 / 2} H_{\beta}^{1 / 2}$ and, hence, $H_{\beta-\varepsilon}^{-1} H_{\beta}^{1 / 2}$ are bounded, the resolvent difference $H_{\beta-\varepsilon}^{-1}-H_{\beta}^{-1}$ is compact. By [7, Theorem 4, Section 1, Chapter 9], we have

$$
\sigma_{\mathrm{ess}}\left(H_{\beta-\varepsilon}\right)=\sigma_{\mathrm{ess}}\left(H_{\beta}\right) .
$$

Remark 4.1. In a completely different context, a second-order perturbation with decaying coefficients which preserves the essential spectrum, has been considered in [3].

Putting together (4.4) and (2.4), we find that

$$
\sigma_{\mathrm{ess}}\left(H_{\beta-\varepsilon}\right)=[\mathcal{E}, \infty)
$$

where $\mathcal{E}$ is defined in (2.5).

\subsection{Main result on the asymptotic distribution of the discrete spectrum of $H_{\beta-\varepsilon}$}

It was shown in [14] that a local decrease of the twisting induces the existence of at least one bound state below $\mathcal{E}$ provided that $\omega$ is not radially symmetric. In other words, 
even if $\varepsilon$ is compactly supported then $H_{\beta-\varepsilon}$ has at least one discrete eigenvalue. Here we show that there is actually an infinite number of bound states appearing below $\mathcal{E}$ if the perturbation $\varepsilon$ decays sufficiently slowly at infinity, and describe the asymptotics as $E \uparrow \mathcal{E}$ of the number of bound states less than $E$.

We will impose on $\varepsilon$ one of the following assumptions:

Assumption 4.2. Suppose that $\varepsilon \in C^{1}(\mathbb{R})$, and there exist constants $\alpha>0$ and $C>0$ such that

$$
\begin{aligned}
0 & \leq \varepsilon(x) \leq C(1+|x|)^{-\alpha}, \\
|\dot{\varepsilon}(x)| & \leq C(1+|x|)^{-\alpha-1}, \quad x \in \mathbb{R} .
\end{aligned}
$$

Assumption 4.3. In addition to Assumption 4.2. suppose that there exists a constant $L>0$ such that

$$
\lim _{|x| \rightarrow \infty}|x|^{\alpha} \varepsilon(x)=L .
$$

In order to formulate our main result, we need the following notations. Let $T$ be a linear self-adjoint operator acting in a given Hilbert space and such that $\tau:=\inf \sigma_{e s s}(T)>$ $-\infty$. Then $N(T ; t), t \in(-\infty, \tau)$, denotes the number of eigenvalues of $T$ lying on the interval $(-\infty, t)$, and counted with the multiplicities.

Theorem 4.4. (i) Suppose that Assumption 4.3 holds with $\alpha \in(0,2)$. Then we have

$$
\lim _{\lambda \downarrow 0} \lambda^{\frac{1}{\alpha}-\frac{1}{2}} N\left(H_{\beta-\varepsilon} ; \mathcal{E}-\lambda\right)=\frac{2}{\pi \alpha \sqrt{\mu}}\left(2 \beta L\left\|\partial_{\varphi} \psi_{1}(\cdot, 0)\right\|_{\mathrm{L}^{2}(\omega)}^{2}\right)^{\frac{1}{\alpha}} B\left(\frac{3}{2}, \frac{1}{\alpha}-\frac{1}{2}\right)
$$

where $\mu$ is defined in (3.8), and $B$ is the Euler beta function.

(ii) Suppose that Assumption 4.3 holds with $\alpha=2$. Then we have

$$
\lim _{\lambda \downarrow 0}|\ln \lambda|^{-1} N\left(H_{\beta-\varepsilon} ; \mathcal{E}-\lambda\right)=\frac{1}{\pi}\left(\frac{2 \beta L}{\mu}\left\|\partial_{\varphi} \psi_{1}(\cdot, 0)\right\|_{L^{2}(\omega)}^{2}-\frac{1}{4}\right)_{+}^{1 / 2} .
$$

If, moreover, $2 \beta L\left\|\partial_{\varphi} \psi_{1}(\cdot, 0)\right\|_{\mathrm{L}^{2}(\omega)}^{2}<\frac{\mu}{4}$, then

$$
N\left(H_{\beta-\varepsilon} ; \mathcal{E}-\lambda\right)=O(1), \quad \lambda \downarrow 0 .
$$

(iii) Suppose that Assumption 4.2 holds with $\alpha>2$. Then we have (4.8).

Remark 4.5. Similarly to the potential perturbation case, the rate of divergence of $N\left(H_{\beta-\varepsilon} ; \mathcal{E}-\lambda\right)$ when $\lambda \downarrow 0$ is determined by the decay rate $\alpha$ of the perturbation. The asymptotic coefficients in (4.6) and (4.7) also depend on the limit $L$, the constant $\beta$, and the geometry of the cross-section $\omega$ through the factor $\left\|\partial_{\varphi} \psi_{1}(\cdot, 0)\right\|_{L^{2}(\omega)}$. Note that if $\left\|\partial_{\varphi} \psi_{1}(\cdot, 0)\right\|_{\mathrm{L}^{2}(\omega)}=0$, then the asymptotic coefficients in (4.6) and (4.7) vanish. However, in this case Proposition 2.2 implies that $\omega$ is a disk centered at the origin. By Remark 2.1, then $H_{\dot{\theta}}$ has purely absolutely continuous spectrum for arbitrary $\theta$, and, in particular, $N\left(H_{\beta-\varepsilon} ; \mathcal{E}-\lambda\right)=0$ for any $\lambda>0$. 


\subsection{Auxiliary results}

This subsection contains some auxiliary results needed for the proof of Theorem 4.4. Let $X_{1}$ and $X_{2}$ be two Hilbert spaces. We denote by $S_{\infty}\left(X_{1}, X_{2}\right)$ the class of linear compact operators $T: X_{1} \rightarrow X_{2}$. If $X_{1}=X_{2}=X$, we write $S_{\infty}(X)$ instead of $S_{\infty}(X, X)$.

Let $T=T^{*} \in S_{\infty}(X)$. Denote by $\mathbb{P}_{J}(T)$ the spectral projection of $T$ associated with the interval $J \subset \mathbb{R}$. For $s>0$ set

$$
n_{ \pm}(s ; T):=\operatorname{rank} \mathbb{P}_{(s, \infty)}( \pm T) .
$$

If $T_{j}=T_{j}^{*} \in S_{\infty}(X), j=1,2$, then the Weyl inequalities

$$
n_{ \pm}\left(s_{1}+s_{2}, T_{1}+T_{2}\right) \leq n_{ \pm}\left(s_{1}, T_{1}\right)+n_{ \pm}\left(s_{2}, T_{2}\right)
$$

hold for $s_{1}>0$ and $s_{2}>0$ (see [6, Chapter I, Eq. (1.31)]).

For $T \in S_{\infty}\left(X_{1}, X_{2}\right)$ put

$$
n_{*}(s ; T):=n_{+}\left(s^{2} ; T^{*} T\right), \quad s>0 .
$$

If $T_{j} \in S_{\infty}\left(X_{1}, X_{2}\right), j=1,2$, then the Ky Fan inequalities

$$
n_{*}\left(s_{1}+s_{2}, T_{1}+T_{2}\right) \leq n_{*}\left(s_{1}, T_{1}\right)+n_{*}\left(s_{2}, T_{2}\right)
$$

hold for $s_{1}>0$ and $s_{2}>0$ (see [6, Chapter I, Eq. (1.32)]).

Denote by $S_{m}\left(X_{1}, X_{2}\right), m \in[1, \infty)$, the Schatten-von Neumann classes of linear compact operators $T: X_{1} \rightarrow X_{2}$ for which the norm $\|T\|_{m}:=\left(\operatorname{Tr}\left(T^{*} T\right)^{m / 2}\right)^{1 / m}$ is finite. If $T \in S_{m}\left(X_{1}, X_{2}\right), m \in[1, \infty)$, then the elementary Chebyshev-type inequality

$$
n_{*}(s ; T) \leq s^{-m}\|T\|_{m}^{m}
$$

holds for any $s>0$.

Lemma 4.6. Let $J \subset \mathbb{R}$. Assume that $G: \mathrm{L}^{2}(J) \rightarrow \mathrm{L}^{2}(\Omega)$ is a bounded operator with integral kernel $g \in \mathrm{L}^{\infty}(\Omega \times J)$. Let $f \in \mathrm{L}^{m}(\Omega), h \in \mathrm{L}^{m}(J)$, with $m \in[2, \infty)$. Then the operator $f G h \in S_{m}\left(\mathrm{~L}^{2}(J), \mathrm{L}^{2}(\Omega)\right)$, and the inequality

$$
\|f G h\|_{m}^{m} \leq C_{m}\|f\|_{\mathrm{L}^{m}(\Omega)}^{m}\|h\|_{\mathrm{L}^{m}(J)}^{m}
$$

holds with $C_{m}=C_{m}(G):=\|G\|^{m-2}\|g\|_{L^{\infty}(\Omega \times J)}^{2}$.

Proof. Assume at first that $f \in \mathrm{L}^{\infty}(\Omega), h \in \mathrm{L}^{\infty}(J)$. Then, evidently, the operator $f G h$ is bounded, and we have

$$
\|f G h\| \leq\|G\|\|f\|_{\mathrm{L}^{\infty}(\Omega)}\|h\|_{\mathrm{L}^{\infty}(J)} .
$$

Assume now that $f \in \mathrm{L}^{2}(\Omega), h \in \mathrm{L}^{2}(J)$. Then $f G h$ is Hilbert-Schmidt, and we have

$$
\|f G h\|_{2} \leq\|g\|_{\mathrm{L}^{\infty}(\Omega \times J)}\|f\|_{\mathrm{L}^{2}(\Omega)}\|h\|_{\mathrm{L}^{2}(J)} .
$$

Applying a standard bilinear interpolation (see [5, Section 4.4]), we get (4.13). 
Remark 4.7. Results similar to Lemma 4.6 are contained in [8] and [23, Lemma 2.3]. We include the proof of the lemma just for the convenience of the reader.

Combining (4.12) with (4.13), we obtain the following

Corollary 4.8. Under the hypotheses of Lemma 4.6 we have

$$
n_{*}(s ; f G h) \leq s^{-m} C_{m}\|f\|_{\mathrm{L}^{m}(\Omega)}^{m}\|h\|_{\mathrm{L}^{m}(J)}^{m}
$$

for each $s>0$.

The following lemma contains standard results on the eigenvalue asymptotics for 1D Schrödinger operators with decaying attractive potentials.

Lemma 4.9. Assume that $V=\bar{V} \in \mathrm{L}^{\infty}(\mathbb{R})$ satisfies

$$
|V(x)| \leq C(1+|x|)^{-\alpha}, \quad x \in \mathbb{R}
$$

with some constants $\alpha>0$ and $C>0$. Let $\hbar>0$, and

$$
\mathcal{H}(\hbar, V):=-\hbar^{2} \frac{d^{2}}{d x^{2}}-V
$$

be the $1 D$ Schrödinger operator with domain $\mathrm{H}^{2}(\mathbb{R})$, self-adjoint in $\mathrm{L}^{2}(\mathbb{R})$.

(i) Assume that $\alpha \in(0,2)$ and there exists a constant $l>0$ such that

$$
\lim _{|x| \rightarrow \infty}|x|^{\alpha} V(x)=l
$$

Then we have

$$
\lim _{\lambda \downarrow 0} \lambda^{\frac{1}{\alpha}-\frac{1}{2}} N(\mathcal{H}(\hbar, V) ;-\lambda)=\frac{2 l^{\frac{1}{\alpha}}}{\pi \alpha \hbar} B\left(\frac{3}{2}, \frac{1}{\alpha}-\frac{1}{2}\right) .
$$

(ii) Assume that (4.16) holds with $\alpha=2$. Then we have

$$
\lim _{\lambda \downarrow 0}|\ln \lambda|^{-1} N(\mathcal{H}(\hbar, V) ;-\lambda)=\frac{1}{\pi}\left(\frac{l}{\hbar^{2}}-\frac{1}{4}\right)_{+}^{1 / 2} .
$$

If, moreover, $l<\frac{\hbar^{2}}{4}$, then

$$
N(\mathcal{H}(\hbar, V) ;-\lambda)=O(1), \quad \lambda \downarrow 0 .
$$

(iii) Suppose that (4.15) holds with $\alpha>2$. Then we have again (4.17).

The first part of the lemma is quite close to [24, Theorem XIII.82], the proof of the second part can be found in [20], while the third part follows from the result of [24, Problem 22, Chapter XIII]. 


\subsection{Proof of Theorem 4.4}

The strategy of the proof of Theorem 4.4 is to reduce the problem of the eigenvalue asymptotics of $H_{\beta-\varepsilon}$ to the one for an effective one-dimensional Schrödinger operator

$$
-\mu \frac{d^{2}}{d x^{2}}-V_{e f f}
$$

where $\mu$ is defined in Corollary [3.3, and the effective potential is given by

$$
V_{\text {eff }}(x)=2 \beta\left\|\partial_{\varphi} \psi_{1}(\cdot ; 0)\right\|_{\mathrm{L}^{2}(\omega)}^{2} \varepsilon(x), \quad x \in \mathbb{R} .
$$

Once this is done, we use Lemma 4.9 to conclude the proof.

The reduction to the one-dimensional problem is rather lengthly and therefore we will divide it in several steps.

\subsubsection{Projection on the bottom of the essential spectrum}

Pick $\delta>0$ so small that the eigenvalue $E_{1}(p)$ is simple for $p \in[-\delta, \delta]$. As explained in at the end of Section 2, we assume that the mappings $[-\delta, \delta] \ni p \mapsto E_{1}(p) \in(0, \infty)$ and $[-\delta, \delta] \ni p \mapsto \psi_{1}(\cdot ; p) \in \mathrm{H}^{2}(\omega)$ are analytic.

Introduce the orthogonal projections

$$
\pi(p):=\left|\psi_{1}(\cdot ; p)\right\rangle\left\langle\psi_{1}(\cdot ; p)\right|, \quad p \in[-\delta, \delta]
$$

acting in $\mathrm{L}^{2}(\omega)$. Denote by $\chi_{\delta}: \mathbb{R} \rightarrow\{0,1\}$ the characteristic function of the interval $(-\delta, \delta)$. Set

$$
\mathcal{P}_{\delta}:=\int_{\mathbb{R}}^{\oplus} \chi_{\delta}(p) \pi(p) d p, \quad P=P_{\delta}:=\mathcal{F}^{*} \mathcal{P}_{\delta} \mathcal{F} .
$$

Evidently, $P$ is an orthogonal projection acting in $\mathrm{L}^{2}(\Omega)$. Put $Q=Q_{\delta}:=I-P_{\delta}$. Since $P$ and $Q$ commute with $H_{\beta}^{-1 / 2}$, they leave $\mathrm{H}_{0}^{1}(\Omega)$ invariant.

Denote by $\mathcal{Z}_{1}(\varepsilon)=\mathcal{Z}_{1}(\varepsilon, \beta, \delta)$ (respectively, $\mathcal{Z}_{2}(\varepsilon)=\mathcal{Z}_{2}(\varepsilon, \beta, \delta)$ ) the self-adjoint operator generated in the Hilbert space $P L^{2}(\Omega)$ (respectively, in $Q L^{2}(\Omega)$ ) by the restriction onto $\mathrm{PH}_{0}^{1}(\Omega)$ (respectively, onto $Q \mathrm{H}_{0}^{1}(\Omega)$ ) of the quadratic form $\mathcal{Q}_{\beta-\varepsilon}$ defined in (2.1). Then the mini-max principle implies

$$
N\left(\mathcal{Z}_{1}(\varepsilon) ; \mathcal{E}-\lambda\right) \leq N\left(H_{\beta-\varepsilon} ; \mathcal{E}-\lambda\right), \quad \lambda>0
$$

Pick $u \in \mathrm{H}_{0}^{1}(\Omega)$ and put $v=P u, w=Q u$ so that $u=v+w$. Then we have

$$
\begin{gathered}
\left\langle W_{\varepsilon, \beta} u, u\right\rangle=: r_{\varepsilon, \beta}[u]=r_{\varepsilon, \beta}[v]+r_{\varepsilon, \beta}[w] \\
-4 \beta \operatorname{Re}\left\langle G_{0} v, G_{0} w\right\rangle-2 \operatorname{Re}\left\langle G_{0} v, G_{1} w\right\rangle-2 \operatorname{Re}\left\langle G_{1} v, G_{0} w\right\rangle+2 \operatorname{Re}\left\langle G_{2} v, G_{2} w\right\rangle
\end{gathered}
$$


where $\langle\cdot, \cdot\rangle$ denotes the scalar product in $\mathrm{L}^{2}(\Omega)$, and $W_{\varepsilon, \beta}$ is the operator defined in (4.3). Next, fix $\nu \in(0,1)$, and on $P D\left(H_{\beta}^{1 / 2}\right)=P L^{2}(\Omega)$ define the operators

$$
G_{3}:=\varepsilon^{(1+\nu) / 2} \partial_{\varphi}^{2}, \quad G_{4}:=\varepsilon^{(1+\nu) / 2} \partial_{\varphi} \partial_{3}, \quad G_{5}:=|\dot{\varepsilon}|^{1 / 2} \partial_{\varphi}, \quad G_{6}:=\varepsilon \partial_{\varphi}^{2} .
$$

Note that the operators $G_{j} P, j=3,4,5,6$, are bounded (and compact) in $\mathrm{L}^{2}(\Omega)$. Integrating by parts, we easily find that

$$
\begin{gathered}
\left\langle G_{0} v, G_{0} w\right\rangle=-\left\langle G_{3} v, \varepsilon^{(1-\nu) / 2} w\right\rangle, \quad\left\langle G_{1} v, G_{0} w\right\rangle=-\left\langle G_{4} v, \varepsilon^{(1-\nu) / 2} w\right\rangle, \\
\left\langle G_{0} v, G_{1} w\right\rangle=-\left\langle G_{4} v, \varepsilon^{(1-\nu) / 2} w\right\rangle-\left\langle G_{5} v, \operatorname{sign} \dot{\varepsilon}|\dot{\varepsilon}|^{1 / 2} w\right\rangle, \quad\left\langle G_{2} v, G_{2} w\right\rangle=-\left\langle G_{6} v, \varepsilon w\right\rangle .
\end{gathered}
$$

Hence, we have

$$
\mathcal{Q}_{\beta-\varepsilon}[u] \geq \mathcal{Q}_{\beta-\varepsilon}[v]-\sum_{j=3}^{6} \int_{\Omega}\left|G_{j} v\right|^{2} d \mathbf{x}+\mathcal{Q}_{\beta-\varepsilon}[w]-\int_{\Omega} \mathcal{V}\left(x_{3}\right)|w(\mathbf{x})|^{2} d \mathbf{x}
$$

where

$$
\mathcal{V}(x):=4\left(\beta^{2}+1\right) \varepsilon(x)^{1-\nu}+|\dot{\varepsilon}(x)|+\varepsilon(x)^{2}, \quad x \in \mathbb{R} .
$$

Denote by $\mathcal{Z}_{1}^{+}(\varepsilon)=\mathcal{Z}_{1}^{+}(\varepsilon, \beta)$ the self-adjoint operator generated in $\operatorname{PL}^{2}(\Omega)$ by the closed quadratic form

$$
\mathcal{Q}_{\beta-\varepsilon}[v]-\sum_{j=3}^{6} \int_{\Omega}\left|G_{j} v\right|^{2} d \mathbf{x}, \quad v \in \operatorname{PH}_{0}^{1}(\Omega) .
$$

Similarly, denote by $\mathcal{Z}_{2}^{+}(\varepsilon)=\mathcal{Z}_{2}^{+}(\varepsilon, \beta)$ the self-adjoint operator generated in $Q \mathrm{~L}^{2}(\Omega)$ by the closed quadratic form

$$
\mathcal{Q}_{\beta-\varepsilon}[w]-\int_{\Omega} \mathcal{V}\left(x_{3}\right)|w(\mathbf{x})|^{2} d \mathbf{x}, \quad w \in Q \mathrm{H}_{0}^{1}(\Omega)
$$

Then, (4.19) implies

$$
N\left(H_{\beta-\varepsilon} ; \mathcal{E}-\lambda\right) \leq N\left(\mathcal{Z}_{1}^{+}(\varepsilon) ; \mathcal{E}-\lambda\right)+N\left(\mathcal{Z}_{2}^{+}(\varepsilon) ; \mathcal{E}-\lambda\right), \quad \lambda>0
$$

Since $\mathcal{V}(x) \rightarrow 0$ as $|x| \rightarrow \infty$, and $\omega$ is a bounded domain, we find that the multiplier by $\mathcal{V}$ is a relative compact perturbation of $H_{\beta-\varepsilon}$. Using this fact and the compactness of the resolvent difference $H_{\beta-\varepsilon}^{-1}-H_{\beta}^{-1}$, we easily check that the difference of the resolvents of the operators $\mathcal{Z}_{2}^{+}(\varepsilon)$ and $\mathcal{Z}_{2}(0)$ is a compact operator. Therefore,

$$
\inf \sigma_{\mathrm{ess}}\left(\mathcal{Z}_{2}^{+}(\varepsilon)\right)=\inf \sigma_{\mathrm{ess}}\left(\mathcal{Z}_{2}(0)\right)=\min \left\{\min _{p \in \mathbb{R}} E_{2}(p), \min _{|p| \geq \delta} E_{1}(p)\right\}>\mathcal{E}
$$

and

$$
N\left(\mathcal{Z}_{2}^{+}(\varepsilon) ; \mathcal{E}-\lambda\right)=O(1), \quad \lambda \downarrow 0,
$$


which combined with (4.20) implies

$$
N\left(H_{\beta-\varepsilon} ; \mathcal{E}-\lambda\right) \leq N\left(\mathcal{Z}_{1}^{+}(\varepsilon) ; \mathcal{E}-\lambda\right)+O(1), \quad \lambda \downarrow 0 .
$$

Fix $\eta \in(0,2 \beta)$. Recalling (4.2) and (4.3), we get

$$
\begin{gathered}
\mathcal{Q}_{\beta-\varepsilon}[v] \leq \mathcal{Q}_{\beta}[v]-(2 \beta-\eta) \int_{\Omega}\left|G_{0} v\right|^{2} d \mathbf{x}+\eta^{-1} \int_{\Omega}\left|G_{1} v\right|^{2} d \mathbf{x}+\int_{\Omega}\left|G_{2} v\right|^{2} d \mathbf{x}, \quad v \in P \mathrm{H}_{0}^{1}(\Omega), \\
\mathcal{Q}_{\beta-\varepsilon}[v]-\sum_{j=3}^{6} \int_{\Omega}\left|G_{j} v\right|^{2} d \mathbf{x} \geq \\
\mathcal{Q}_{\beta}[v]-(2 \beta+\eta) \int_{\Omega}\left|G_{0} v\right|^{2} d \mathbf{x}-\eta^{-1} \int_{\Omega}\left|G_{1} v\right|^{2} d \mathbf{x}-\sum_{j=3}^{6} \int_{\Omega}\left|G_{j} v\right|^{2} d \mathbf{x}, \quad v \in P \mathrm{H}_{0}^{1}(\Omega) .
\end{gathered}
$$

Denote by $\tilde{\mathcal{Z}}_{1}^{-}(\varepsilon)=\tilde{\mathcal{Z}}_{1}^{-}(\varepsilon, \beta, \eta)$ the self-adjoint operator generated in $\operatorname{PL}^{2}(\Omega)$ by the closed quadratic form

$$
\mathcal{Q}_{\beta}[v]-(2 \beta-\eta) \int_{\Omega}\left|G_{0} v\right|^{2} d \mathbf{x}+\eta^{-1} \int_{\Omega}\left|G_{1} v\right|^{2} d \mathbf{x}+\int_{\Omega}\left|G_{2} v\right|^{2} d \mathbf{x}, \quad v \in \mathrm{PH}_{0}^{1}(\Omega) .
$$

Similarly, denote by $\tilde{\mathcal{Z}}_{1}^{+}(\varepsilon)=\tilde{\mathcal{Z}}_{1}^{+}(\varepsilon, \beta, \eta)$ the self-adjoint operator generated in $P \mathrm{~L}_{2}(\Omega)$ by the closed quadratic form

$$
\mathcal{Q}_{\beta}[v]-(2 \beta+\eta) \int_{\Omega}\left|G_{0} v\right|^{2} d \mathbf{x}-\eta^{-1} \int_{\Omega}\left|G_{1} v\right|^{2} d \mathbf{x}-\sum_{j=3}^{6} \int_{\Omega}\left|G_{j} v\right|^{2} d \mathbf{x}, \quad v \in P \mathrm{H}_{0}^{1}(\Omega) .
$$

Then (4.22) - (4.23) implies

$$
\mathcal{Z}_{1}^{+} \geq \tilde{\mathcal{Z}}_{1}^{+}, \quad \mathcal{Z}_{1} \leq \tilde{\mathcal{Z}}_{1}^{-} .
$$

Now estimates (4.18), (4.21), and (4.24) entail

$$
N\left(\tilde{\mathcal{Z}}_{1}^{-}-\mathcal{E}+\lambda ; 0\right) \leq N\left(H_{\beta-\varepsilon} ; \mathcal{E}-\lambda\right) \leq N\left(\tilde{\mathcal{Z}}_{1}^{+}-\mathcal{E}+\lambda ; 0\right)+O(1), \quad \lambda \downarrow 0 .
$$

The last equation shows that the eigenvalue asymptotics of $H_{\beta-\varepsilon}$ is determined by the asymptotics of the reduced operator $\mathrm{PH}_{\beta-\varepsilon} P$ modulo some error terms.

\subsubsection{Reduction to a one-dimensional problem}

We introduce the operator $U: \mathrm{L}^{2}(-\delta, \delta) \rightarrow P \mathrm{~L}^{2}(\Omega)$ which acts on $f \in \mathrm{L}^{2}(-\delta, \delta)$ as follows

$$
(U f)\left(x_{t}, x_{3}\right):=\mathcal{F}^{*} \tilde{f}, \quad \tilde{f}\left(x_{t}, p\right):=\left\{\begin{array}{l}
\psi_{1}\left(x_{t}, p\right) f(p) \quad \text { if } \quad x_{t} \in \omega, p \in(-\delta, \delta), \\
0 \quad \text { if } \quad x_{t} \in \omega, p \in \mathbb{R} \backslash(-\delta, \delta) .
\end{array}\right.
$$


Then $U$ is a unitary operator and using (4.26) it can be directly verified that

$$
\begin{gathered}
\tilde{\mathcal{Z}}_{1}^{-}-\mathcal{E}+\lambda=U\left(M(\lambda)-(2 \beta-\eta) \Gamma_{0}^{*} \Gamma_{0}+\eta^{-1} \Gamma_{1}^{*} \Gamma_{1}+\Gamma_{2}^{*} \Gamma_{2}\right) U^{*}, \\
\tilde{\mathcal{Z}}_{1}^{+}-\mathcal{E}+\lambda=U\left(M(\lambda)-(2 \beta+\eta) \Gamma_{0}^{*} \Gamma_{0}-\eta^{-1} \Gamma_{1}^{*} \Gamma_{1}-\sum_{j=3}^{6} \Gamma_{j}^{*} \Gamma_{j}\right) U^{*},
\end{gathered}
$$

where $M(\lambda)$ is the multiplier by $E_{1}(p)-\mathcal{E}+\lambda$ in $\mathrm{L}^{2}(-\delta, \delta)$, and $\Gamma_{j}: \mathrm{L}^{2}(-\delta, \delta) \rightarrow \mathrm{L}^{2}(\Omega)$ are integral operators with kernels $(2 \pi)^{-1 / 2} e^{i x_{3} p} \gamma_{j}(\mathbf{x}, p), \mathbf{x}=\left(x_{t}, x_{3}\right) \in \Omega, p \in(-\delta, \delta)$, defined by

$$
\begin{gathered}
\gamma_{0}(\mathbf{x}, p):=\varepsilon\left(x_{3}\right)^{1 / 2} \partial_{\varphi} \psi_{1}\left(x_{t} ; p\right), \quad \gamma_{1}(\mathbf{x}, p):=i \varepsilon\left(x_{3}\right)^{1 / 2} \psi_{1}\left(x_{t} ; p\right) p \\
\gamma_{2}(\mathbf{x}, p):=\varepsilon\left(x_{3}\right) \partial_{\varphi} \psi_{1}\left(x_{t} ; p\right), \quad \gamma_{3}(\mathbf{x}, p):=\varepsilon\left(x_{3}\right)^{(1+\nu) / 2} \partial_{\varphi}^{2} \psi_{1}\left(x_{t} ; p\right), \\
\gamma_{4}(\mathbf{x}, p):=i \varepsilon\left(x_{3}\right)^{(1+\nu) / 2} \partial_{\varphi} \psi_{1}\left(x_{t} ; p\right) p, \quad \gamma_{5}(\mathbf{x}, p):=\left|\dot{\varepsilon}\left(x_{3}\right)\right|^{1 / 2} \partial_{\varphi} \psi_{1}\left(x_{t} ; p\right), \\
\gamma_{6}(\mathbf{x}, p):=\varepsilon\left(x_{3}\right) \partial_{\varphi}^{2} \psi_{1}\left(x_{t} ; p\right) .
\end{gathered}
$$

Then (4.25) implies

$$
\begin{gathered}
N\left(M(\lambda)-(2 \beta-\eta) \Gamma_{0}^{*} \Gamma_{0}+\eta^{-1} \Gamma_{1}^{*} \Gamma_{1}+\Gamma_{2}^{*} \Gamma_{2} ; 0\right) \leq \\
N\left(H_{\beta-\varepsilon} ; \mathcal{E}-\lambda\right) \leq \\
N\left(M(\lambda)-(2 \beta+\eta) \Gamma_{0}^{*} \Gamma_{0}-\eta^{-1} \Gamma_{1}^{*} \Gamma_{1}-\sum_{j=3}^{6} \Gamma_{j}^{*} \Gamma_{j} ; 0\right)+O(1), \quad \lambda \downarrow 0 .
\end{gathered}
$$

Set

$$
a_{\lambda}(p):=\left(E_{1}(p)-\mathcal{E}+\lambda\right)^{-1 / 2}, \quad \lambda>0, \quad p \in[-\delta, \delta] .
$$

Applying the Birman-Schwinger principle, the Weyl inequalities (4.9), and definition (4.10), we find that for each $s \in(0,1)$ we have

$$
\begin{gathered}
N\left(M(\lambda)-(2 \beta-\eta) \Gamma_{0}^{*} \Gamma_{0}+\eta^{-1} \Gamma_{1}^{*} \Gamma_{1}+\Gamma_{2}^{*} \Gamma_{2} ; 0\right)= \\
n_{+}\left(1 ; a_{\lambda}\left((2 \beta-\eta) \Gamma_{0}^{*} \Gamma_{0}-\eta^{-1} \Gamma_{1}^{*} \Gamma_{1}-\Gamma_{2}^{*} \Gamma_{2}\right) a_{\lambda}\right) \geq \\
n_{*}\left(\sqrt{(1+s) /(2 \beta-\eta)} ; \Gamma_{0} a_{\lambda}\right)-n_{*}\left(\sqrt{\eta s / 2} ; \Gamma_{1} a_{\lambda}\right)-n_{*}\left(\sqrt{s / 2} ; \Gamma_{2} a_{\lambda}\right), \\
N\left(M(\lambda)-(2 \beta+\eta) \Gamma_{0}^{*} \Gamma_{0}-\eta^{-1} \Gamma_{1}^{*} \Gamma_{1}-\sum_{j=3}^{6} \Gamma_{j}^{*} \Gamma_{j} ; 0\right)= \\
n_{+}\left(1 ; a_{\lambda}\left((2 \beta+\eta) \Gamma_{0}^{*} \Gamma_{0}+\eta^{-1} \Gamma_{1}^{*} \Gamma_{1}+\sum_{j=3}^{6} \Gamma_{1}^{*} \Gamma_{1}\right) a_{\lambda}\right) \leq
\end{gathered}
$$




$$
n_{*}\left(\sqrt{(1-s) /(2 \beta+\eta)} ; \Gamma_{0} a_{\lambda}\right)+n_{*}\left(\sqrt{\eta s / 5} ; \Gamma_{1} a_{\lambda}\right)+\sum_{j=3}^{6} n_{*}\left(\sqrt{s / 5} ; \Gamma_{j} a_{\lambda}\right) .
$$

Note that on the right-hand sides of (4.28) and (4.29) there are just linear combinations of terms of the form $n_{*}\left(r ; \Gamma_{j} a_{\lambda}\right)$ with $r>0$ independent of $\lambda$ and $j=0, \ldots, 6$. The rest of the proof of Theorem 4.4 reduces to the asymptotic analysis as $\lambda \downarrow 0$ of these terms. Our aim is to show that only the ones corresponding to the operator $\Gamma_{0}$ contribute to the main asymptotic term of $N\left(H_{\beta-\varepsilon} ; \mathcal{E}-\lambda\right)$ as $\lambda \downarrow 0$. First, we show that

$$
n_{*}\left(r ; \Gamma_{j} a_{\lambda}\right)=O(1), \quad \lambda \downarrow 0, \quad j=1,4,
$$

for every $r>0$. To this end, it suffices to apply (4.14) with $J=(-\delta, \delta), f(\mathbf{x})=\varepsilon\left(x_{3}\right)^{1 / 2}$ if $j=1, f(\mathbf{x})=\varepsilon\left(x_{3}\right)^{(1+\nu) / 2}$ if $j=4, g(\mathbf{x}, p)=(2 \pi)^{-1 / 2} e^{i x_{3} p} \partial_{\varphi} \psi_{1}\left(x_{t} ; p\right), h(p)=i p a_{\lambda}(p)$, and $m \in[2, \infty)$ large enough.

Further, for $j=0,2,3,5,6$ we define the operators $\tilde{\Gamma}_{j}: \mathrm{L}^{2}(\mathbb{R}) \rightarrow \mathrm{L}^{2}(\Omega)$ as integral operators whose kernels are obtained by substituting $\psi_{1}\left(x_{t} ; 0\right)$ for $\psi_{1}\left(x_{t} ; p\right)$ in the expressions for the integral kernels of $\Gamma_{j}$. Denote by $\tilde{\Gamma}_{j, \delta}$ the restriction of $\tilde{\Gamma}_{j}$ onto $L^{2}(-\delta, \delta)$. Set

$$
\tilde{a}_{\lambda}(p)=\left(\mu p^{2}+\lambda\right)^{-1 / 2}, \quad p \in \mathbb{R}, \quad \lambda>0 .
$$

Pick $s \in(0,1)$, and bearing in mind (3.9) choose $\delta>0$ so small that we have

$$
(1+s)^{-1} \tilde{a}_{\lambda}(p) \leq a_{\lambda}(p) \leq(1-s)^{-1} \tilde{a}_{\lambda}(p), \quad p \in[-\delta, \delta], \quad \lambda>0 .
$$

Our next goal is to show that the quantities $n_{*}\left(r ; \Gamma_{j} a_{\lambda}\right), r>0, j=2,3,5,6$, appearing at the right-hand sides of (4.28) and (4.29) are bounded under the hypotheses of Theorem 4.4 (ii) - (iii), and do not contribute to the main asymptotic term as $\lambda \downarrow 0$ of $N\left(H_{\beta-\varepsilon} ; \mathcal{E}-\right.$ $\lambda)$ under the hypotheses of Theorem 4.4 (i), even though in the last case they might not be bounded in contrast to the cases $j=1,4$. The upper bound in (4.31) combined with the mini-max principle, and the Ky Fan inequalities (4.11) imply

$$
n_{*}\left(r ; \Gamma_{j} a_{\lambda}\right) \leq n_{*}\left(r(1-s)^{2} ; \tilde{\Gamma}_{j} \tilde{a}_{\lambda}\right)+n_{*}\left(r s ;\left(\Gamma_{j}-\tilde{\Gamma}_{j, \delta}\right) a_{\lambda}\right), \quad r>0, \quad j=2,3,5,6 .
$$

It is quite easy to see that

$$
n_{*}\left(r s ;\left(\Gamma_{j}-\tilde{\Gamma}_{j, \delta}\right) a_{\lambda}\right)=O(1), \quad \lambda \downarrow 0,
$$

for $r>0$ and $j=2,3,5,6$; for example, if $j=2$ it suffices to apply (4.14) with $f(\mathbf{x})=\varepsilon\left(x_{3}\right), g(\mathbf{x}, p)=(2 \pi)^{-1 / 2} e^{i x_{3} p} p^{-1}\left(\partial_{\varphi} \psi_{1}\left(x_{t} ; p\right)-\partial_{\varphi} \psi_{1}\left(x_{t} ; 0\right)\right), h(p)=a_{\lambda}(p) p$, and $m \in[2, \infty)$ large enough, and if $j=3,5,6$, the argument is the same with appropriate choice of $f, g$, and $h$.

Now, the Birman-Schwinger principle implies that for each $r>0$ we have

$$
n_{*}\left(r ; \tilde{\Gamma}_{j} \tilde{a}_{\lambda}\right)=n_{+}\left(r^{2} ; \tilde{a}_{\lambda} \tilde{\Gamma}_{j}^{*} \tilde{\Gamma}_{j} \tilde{a}_{\lambda}\right)=N\left(\mathcal{H}\left(\sqrt{\mu}, r^{-2} V_{j}\right) ;-\lambda\right), \quad j=2,3,5,6, \quad \lambda>0,
$$


where $\mathcal{H}$ is the $1 \mathrm{D}$ Schrödinger operator defined in Lemma 4.9, and

$$
\begin{gathered}
V_{2}(x):=\left\|\partial_{\varphi} \psi_{1}(\cdot ; 0)\right\|_{\mathrm{L}^{2}(\omega)}^{2} \varepsilon(x)^{2}, \quad V_{3}(x):=\left\|\partial_{\varphi}^{2} \psi_{1}(\cdot ; 0)\right\|_{\mathrm{L}^{2}(\omega)}^{2} \varepsilon(x)^{1+\nu}, \\
V_{5}(x):=\left\|\partial_{\varphi} \psi_{1}(\cdot ; 0)\right\|_{\mathrm{L}^{2}(\omega)}^{2}|\dot{\varepsilon}(x)|, \quad V_{6}(x):=\left\|\partial_{\varphi}^{2} \psi_{1}(\cdot ; 0)\right\|_{\mathrm{L}^{2}(\omega)}^{2} \varepsilon(x)^{2}, \quad x \in \mathbb{R} .
\end{gathered}
$$

Assumption 4.2 implies that $\lim _{|x| \rightarrow \infty}|x|^{\alpha} V_{j}(x)=0, j=2,3,5,6$. Applying Lemma 4.9 to the counting function $N\left(\mathcal{H}\left(\sqrt{\mu}, r^{-2} V_{j}\right) ;-\lambda\right)$, and putting together (4.32) - (4.34), we obtain that for $j=2,3,5,6$, and $r>0$, we have

$$
n_{*}\left(r ; \Gamma_{j} a_{\lambda}\right)=\left\{\begin{array}{l}
o\left(\lambda^{\frac{1}{2}-\frac{1}{\alpha}}\right) \text { if Assumption } 4.3 \text { holds with } \alpha \in(0,2), \\
O(1)=o(|\ln \lambda|) \text { if Assumption } 4.3 \text { holds with } \alpha=2, \\
O(1) \text { if Assumption } 4.2 \text { holds with } \alpha>2,
\end{array}\right.
$$

as $\lambda \downarrow 0$.

\subsubsection{Eigenvalue asymptotics for the one-dimensional operator}

Finally, we will show that the quantities $n_{*}\left(r ; \Gamma_{0} a_{\lambda}\right), r>0$, on the right-hand sides of (4.28) and (4.29), generate the main asymptotic term as $\lambda \downarrow 0$ of $N\left(H_{\beta-\varepsilon} ; \mathcal{E}-\lambda\right)$ under the hypotheses of Theorem 4.4 (i) - (ii), or are bounded under the hypotheses of Theorem 4.4 (iii). Similarly to (4.32), the estimates (4.31), combined with the mini-max principle, and the Ky Fan inequalities (4.11) imply

$$
\begin{aligned}
& n_{*}\left(r(1+s)^{3} ; \tilde{\Gamma}_{0} \tilde{a}_{\lambda}\right)-n_{*}\left(r s(1+s)^{2} ; \tilde{\Gamma}_{0} \tilde{a}_{\lambda} w_{\delta}\right)-n_{*}\left(r s ;\left(\Gamma_{0}-\tilde{\Gamma}_{0, \delta}\right) a_{\lambda}\right) \leq \\
& n_{*}\left(r ; \Gamma_{0} a_{\lambda}\right) \leq n_{*}\left(r(1-s)^{2} ; \tilde{\Gamma}_{0} \tilde{a}_{\lambda}\right)+n_{*}\left(r s ;\left(\Gamma_{0}-\tilde{\Gamma}_{0, \delta}\right) a_{\lambda}\right), \quad r>0,
\end{aligned}
$$

where $w_{\delta}: \mathbb{R} \rightarrow\{0,1\}$ denotes the characteristic function of the set $\mathbb{R} \backslash(-\delta, \delta)$.

By analogy with (4.33), we find that

$$
n_{+}\left(r ;\left(\Gamma_{0}-\tilde{\Gamma}_{0, \delta}\right) a_{\lambda}\right)=O(1), \quad \lambda \downarrow 0, \quad r>0 .
$$

Further, applying (4.14) with

$$
J=\mathbb{R}, \quad f(\mathbf{x})=\varepsilon\left(x_{3}\right)^{1 / 2}, \quad g(\mathbf{x}, p)=(2 \pi)^{-1 / 2} e^{i x_{3} p} \partial_{\varphi} \psi_{1}\left(x_{t} ; 0\right), \quad h(p)=a_{\lambda}(p) w_{\delta}(p),
$$

and $m \in[2, \infty)$ large enough, we get

$$
n_{*}\left(r ; \tilde{\Gamma}_{0} \tilde{a}_{\lambda} w_{\delta}\right)=O(1), \quad \lambda \downarrow 0, \quad r>0 .
$$

The Birman-Schwinger principle implies that for each $r>0$ we have

$$
n_{*}\left(r ; \tilde{\Gamma}_{0} \tilde{a}_{\lambda}\right)=n_{+}\left(r^{2} ; \tilde{a}_{\lambda} \tilde{\Gamma}_{0}^{*} \tilde{\Gamma}_{0} \tilde{a}_{\lambda}\right)=N\left(\mathcal{H}\left(\sqrt{\mu}, r^{-2} V_{0}\right) ;-\lambda\right), \quad \lambda>0,
$$

where $\mathcal{H}$ is the $1 \mathrm{D}$ Schrödinger operator defined in Lemma 4.9, and

$$
V_{0}(x):=\left\|\partial_{\varphi} \psi_{1}(\cdot ; 0)\right\|_{L^{2}(\omega)}^{2} \varepsilon(x) .
$$


Applying Lemma 4.9, and bearing in mind (4.36) - (4.38), we find that

$$
\lim _{\lambda \downarrow 0} \lambda^{\frac{1}{\alpha}-\frac{1}{2}} n_{*}\left(r ; \Gamma_{0} a_{\lambda}\right)=\frac{2\left(r^{-2}\left\|\partial_{\varphi} \psi_{1}(\cdot ; 0)\right\|_{L^{2}(\omega)}^{2} L\right)^{\frac{1}{\alpha}}}{\pi \alpha \sqrt{\mu}} B\left(\frac{3}{2}, \frac{1}{\alpha}-\frac{1}{2}\right) .
$$

if Assumption 4.3 with $\alpha \in(0,2)$ holds true,

$$
\lim _{\lambda \downarrow 0}|\ln \lambda|^{-1} n_{*}\left(r ; \Gamma_{0} a_{\lambda}\right)=\frac{1}{\pi}\left(\frac{r^{-2}\left\|\partial_{\varphi} \psi_{1}(\cdot ; 0)\right\|_{\mathrm{L}^{2}(\omega)}^{2} L}{\mu}-\frac{1}{4}\right)_{+}^{1 / 2},
$$

if Assumption 4.3 with $\alpha=2$ holds true, and

$$
n_{*}\left(r ; \Gamma_{0} a_{\lambda}\right)=O(1), \quad \lambda \downarrow 0,
$$

if Assumption 4.2 holds true with $\alpha>2$. If, moreover, Assumption 4.3 holds with $\alpha=2$, and $r^{-2}\left\|\partial_{\varphi} \psi_{1}(\cdot ; 0)\right\|_{\mathrm{L}^{2}(\omega)}^{2} L<\frac{\mu}{4}$, then we have (4.42).

Since the numbers $s \in(0,1)$ and $\eta \in(0,2 \beta)$ in (4.28) - (4.29) could be chosen arbitrarily small, we find that (4.27) - (4.29), (4.35), (4.36), (4.40) - (4.42) imply that under the appropriate assumptions of Theorem 4.4, asymptotic relations (4.6), (4.7), or (4.8) hold true.

Acknowledgements. Hynek Kovařík was partially supported by the German Research Foundation (DFG) under Grant KO 3636/1-1. Georgi Raikov was partially supported by the Chilean Scientific Foundation Fondecyt under Grant 1050716.

\section{References}

[1] M.Abramowitz, I.Stegun, Handbook of Mathematical Functions with Formulas, Graphs, and Mathematical Tables, National Bureau of Standards, Applied Mathematics Series 55, 1964.

[2] S. Agmon, Lectures on Elliptic Boundary Value Problems, Van Nostrand Co., Inc., Princeton, N.J.-Toronto-London 1965.

[3] S. Alama, M. Avellaneda, P. A. Deift, R. Hempel, On the existence of eigenvalues of a divergence-form operator $A+\lambda B$ in a gap of $\sigma(A)$, Asymptotic Anal. 8 (1994), 311-344.

[4] G. Allaire, A. Piatnitski, Homogenization of the Schrödinger equation and effective mass theorems, Comm. Math. Phys. 258 (2005), 1-22.

[5] J. Bergh, J. LÖFström, Interpolation Spaces. An Introduction, Grundlehren der Mathematischen Wissenschaften, 223, Springer-Verlag, Berlin-HeidelbergNew York, 1976. 
[6] M.S̆.BIRman, M.Z.Solomjak, Quantitative analysis in Sobolev imbedding theorems and applications to spectral theory, Amercan Math. Society Translations Series 2, 114, AMS, Providence R.I., 1980.

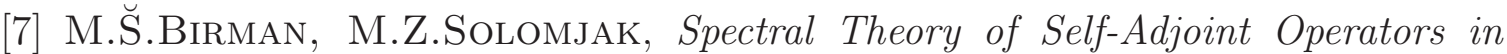
Hilbert Space, Reidel Publishing Company, Dordrecht, 1987.

[8] M.S. Birman, M.Z. Solomyak, Schrödinger Operator. Estimates for number of bound states as function-theoretical problem, In: Spectral theory of operators (Novgorod, 1989), 1-54, Amer. Math. Soc. Transl. Ser. 2, 150, Amer. Math. Soc., Providence, RI, 1992.

[9] M. Sh. Birman, T. A. Suslina, Periodic second-order differential operators. Threshold properties and averaging,(Russian) Algebra i Analiz 15 (2003), 1-108; English translation in St. Petersburg Math. J. 15 (2004), 639-714.

[10] G. Bouchitté, M. L. Mascarenhas, L. Trabucho, On the curvarture and torsion effects in one dimensional waveguides, Control, Optim. Calc. Var. 13 (2007), 793-808.

[11] Ph. Briet, G. Raikov, E. Soccorsi, Spectral properties of a magnetic quantum Hamiltonian in a strip, Asympt. Anal. 58 (2008), 127-155.

[12] T. Ekholm, H. Kovařík, Stability of the magnetic Schrödinger operator in a waveguide, Comm. Partial Differential Equations 30, (2005) 539-565.

[13] T. Ekholm, H. KovaŘÍK, D. KRejčiřík, A Hardy inequality in twisted waveguides, Arch. Ration. Mech. Anal. 188 (2008), 245-264.

[14] P. Exner, H. KovaříK, Spectrum of the Schrödinger operator in a perturbed periodically twisted tube, Lett. Math. Phys. 73 (2005) 183-192.

[15] V. Geyler, M. Senatorov, The structure of the spectrum of the Schrödinger operator with a magnetic field in a strip, and finite-gap potentials, Mat.Sb. 188 (1997), 21-.32 (Russian); English translation in Sb.Math. 188 (1997), 657-669.

[16] D. Gilbarg, N. S. Trudinger, Elliptic Partial Differential Equations of Second Order, Classics in Mathematics, Springer-Verlag New York, Inc., New York 1998.

[17] D. Jerison, C. E. Kenig, Unique continuation and absence of positive eigenvalues for Schrödinger operators, Ann. of Math. (2) 121 (1985), 463-494.

[18] T. Kato, Perturbation Theory for Linear Operators, Die Grundlehren der mathematischen Wissenschaften, 132 Springer-Verlag New York, Inc., New York 1966.

[19] W. Kinsch, B. Simon, Comparison theorems for the gap of Schrödinger operators, J. Funct. Anal. 75 (1987), 396-410.

[20] W. Kirsch, B. Simon, Corrections to the classical behavior of the number of bound states of Schrödinger operators, Ann. Physics 183 (1988), 122-130. 
[21] D. KREJČIř́K, Twisting versus bending in quantum waveguides, Proceedings of the research programme "Analysis on graphs and applications", Isaac Newton Institute (Cambridge), to appear.

[22] B. M. Levitan, I. S. Sargsyan, Introduction to Spectral Theory. Selfadjoint Ordinary Differential Operators, (Russian) Nauka, Moscow, 1970.

[23] G. RAIKOv, Eigenvalue asymptotics for the Schrödinger operator with perturbed periodic potential, Invent. Math. 110 (1992), 75-93.

[24] M. Reed, B. Simon, Methods of Modern Mathematical Physics IV: Analysis of Operators, Academic Press, 1978.

Ph. BRIET

Centre de Physique Théorique

CNRS-Luminy, Case 907

13288 Marseille, France

E-mail: briet@cpt.univ-mrs.fr

\section{H. KOVAŘÍK}

Università degli Studi di Modena e Reggio Emilia

Dipartimento di Matematica

Via Campi 213/B

I-41100 Modena, Italy

E-mail: hynek.kovarik@unimore.it

\section{G. RAIKOV}

Facultad de Matemáticas

Pontificia Universidad Católica de Chile

Av. Vicuña Mackenna 4860

Santiago de Chile

E-mail: graikov@mat.puc.cl

\section{E. SOCCORSI}

Centre de Physique Théorique

CNRS-Luminy, Case 907

13288 Marseille, France

E-mail: soccorsi@cpt.univ-mrs.fr 